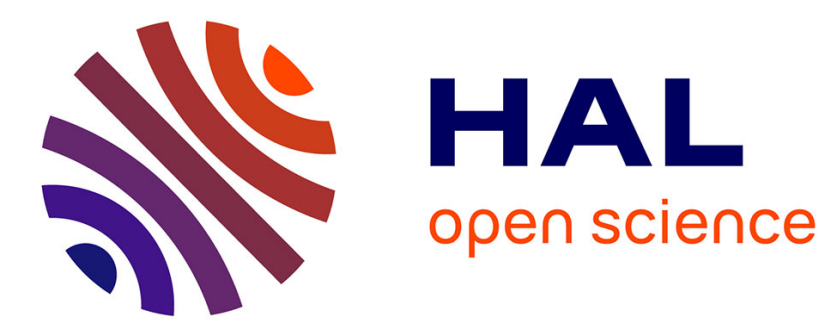

\title{
Map-Aided Localization with Lateral Perception
}

Dominique Gruyer, Rachid Belaroussi, Marc Revilloud

\section{To cite this version:}

Dominique Gruyer, Rachid Belaroussi, Marc Revilloud. Map-Aided Localization with Lateral Perception. IEEE Intelligent Vehicles Symposium, Jun 2014, France. 7p. hal-01056258

\section{HAL Id: hal-01056258 https://hal.science/hal-01056258}

Submitted on 18 Aug 2014

HAL is a multi-disciplinary open access archive for the deposit and dissemination of scientific research documents, whether they are published or not. The documents may come from teaching and research institutions in France or abroad, or from public or private research centers.
L'archive ouverte pluridisciplinaire HAL, est destinée au dépôt et à la diffusion de documents scientifiques de niveau recherche, publiés ou non, émanant des établissements d'enseignement et de recherche français ou étrangers, des laboratoires publics ou privés. 


\title{
Map-Aided Localization with Lateral Perception
}

\author{
Dominique Gruyer, Rachid Belaroussi, and Marc Revilloud
}

\begin{abstract}
Accurate localization of a vehicle is a challenging task as GPS available on the market are not designed for lane-level accuracy application. Although dead reckoning helps, cumulative errors from inertial sensors result in a integration drift. This paper presents a new method of localization based on sensors data fusion. An accurate digital map of the lane marking is used as a powerful additional sensor. Road markings are detected by processing two lateral cameras to estimate their distance to the vehicle. Coupled with the map data in a EKF filter it improves the ego-localization obtained with inertial and GPS measurements. The result is a vehicle localization at an ego-lane level of accuracy, with a lateral error of less than $\mathbf{1 0}$ centimeters.
\end{abstract}

\section{INTRODUCTION}

Driver Assistance Systems developed over the last decade have required a precise and robust estimation of road scene major features. Those features include obstacles (vehicle, pedestrian), road (marking, lanes, traffic signs), and the egovehicle (localization and dynamics of the vehicle). Usually each feature is addressed separately, for instance obstacle detection in collision avoidance, road attributes in lane keeping assistance, ego-localization in navigation systems. Recently, automated driving systems have made it necessary to fuse the attributes of different features to obtain more precise, robust and complete information.

In the frame of french, european and international projects (respectively ABV, eFuture, CooPerCom) we have tackled the task of perception of the environment including the attributes of these three features. More specifically, we develop an application localizing the ego-vehicle in its lane allowing a positioning and a lateral control precise enough to be applicable.

There is a body of work in the field of robust localization by hybrid data fusion (proprio and exteroceptive): monomodel approaches (EKF, UKF, DD1, DD2) [1], [2], [3], multi-model [4], [5] and particular filter [6] achieve localization with a precision to the meter. For instance, in [5], the approach is only centered on the ego-vehicle but can compute the likelihood of each model to build the finale estimation. In [6] a map is used to filter out the particles out of a road. In this case, the localization process converges rapidly toward a solution inside a roadway. However it cannot specify which lane the vehicle is on.

It is clear that the use of a geographic map can be an advantage: commercial navigation systems routinely operate MapMatching by coupling maps and vehicle positioning. In [7] a map-matching algorithm using the visual odometry motion trajectory estimation (from a stereovision rig) as input

Authors are with IFSTTAR, COSYS-LIVIC, 77 rue des chantiers, F78000, Versailles, France, e-mail: dominique.gruyer@ifsttar.fr
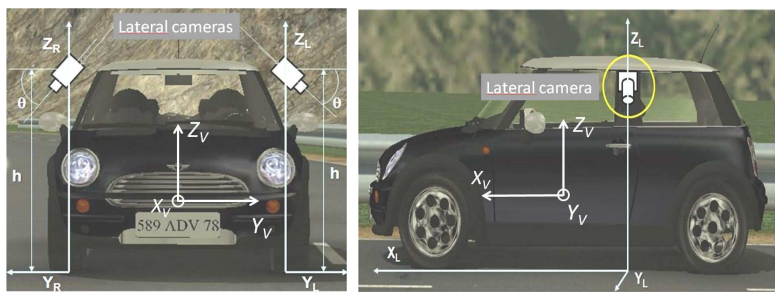

Fig. 1. Extrinsic configuration of the side cameras and associated coordinate systems.

and corrected using the digital map features, greatly improve the global localization performance. In [8], a standard navigation map is matched with a laser scanner occupancy grid map, a video based grid map and a lane marking grid map for road course prediction. Alternately, in [9] a radar local map is built by estimating the ego-motion from odomotetry measurements with an EKF. Matching the digital road map and the grid maps is optimized with a Levenberg-Marquardt or a particle filter.

Recent approaches assume the road network has been surveyed accurately beforehand. In [10] a loosely coupled GPS/INS system is used with a camera: the digital map is made of polylines and area information divided into three classes (line landmark, road surface and background) which are projected in the image plane. Appearance of these classes are modeled by the distribution of their coherency values, and used in a particle filter. In [11] the absolute position of corners of ground markings (such as arrows, speed limit, or texts) were precisely surveyed and are matched to corners detected by FAST with a camera in order to estimate its pose and location. In [12], a vehicle with a backward facing camera is used to create a 3D landmarks map of the environment. Landmarks are matched into the current image and back projection errors are minimized yielding a rough single shot pose estimate. IMU measurements are blended with past single shot estimates yielding the final ego-pose.

In order to enhance accuracy and potentially to determine the traffic lane being travelled, [13], [4] proposed to use a map of the lane marking with a centimeter level accuracy coupled with a differential GPS EGNOS and inertial datas. The combination is done by way of a particle filter. The map is used as a geometrical constraint in the ego-positioning of the vehicle and for map-matching purposes. However, the lack of GPS data or in case of multipath effect, this approach approach is not able to function properly and to guaranty the integrity of its results. A relevant solution is to add a local processing providing a lane marking detection. It can provide a more accurate localization of the vehicle inside its lane. 
Recently, [1] proposed an EKF-based algorithm fusing GPS, IMU and lane marking information: they have shown that the use of visual features can improve the lateral localization up to a centimeter-level accuracy (less than $30 \mathrm{~cm}$ ). Their experimental setup uses one camera directed frontward to detect lane marking and estimate their lateral distance to the vehicle. In [14] a similar setup (GPS, IMU, front camera) is used with the addition of a laser scanner, the digital map also containing location of landmarks such as traffic signs, tree or guide post. Extracted features (lane markings and landmarks) are associated with the elements in the digital map to correct the pose estimation which is roughly initialized with GPS. A particle filter is to implement the ego-localization algorithm.

In [15], a method to improve global localization in an intersection is based on the alignement of visual landmarks with the information from an extended digital map. A stereovision system provides a detailed 3D perception of road landmarks such as lateral lane delimiter, painted traffic signs, curbs and stop lines. Combination of visual and enriched map of the intersection is done with a Bayesian network, yielding to a global localization with a submeter level of accuracy.

In this paper, we propose an algorithm that fuses localization data, road marking detection and a digital map of the road (location of the edges of the left and right lanes) in order to obtain a centimeter lateral localization of our vehicle. Our approach uses two lateral cameras detecting left and right markings independently, with a focus on lane segment close to the vehicle leading. We believe this setup improves the lateral localization and is specific enough for the vehicle to be considered as moving on rails.

\section{In-Vehicle Apparatus}

The experimental setup uses two cameras positioned on each side of the vehicle and directed toward the ground. In this configuration, cameras can be low cost and have a low resolution because the scope of information to be extracted is only a little over a meter. This configuration is shown in Figure 1 Several coordinate frames have to be considered: for each sensor, there is the image frame $(u, v)$ and the camera frame to be able to describe the characteristic of a road mark in respect of each camera.

The other sensors considered are proprioceptive sensors:

- a GPS-receiver with a slow refresh rate $(1 \mathrm{~Hz})$ : it provides vehicle absolute position in the world coordinate frame $\mathcal{R}_{0}$,

- an Inertial Navigation System INS (66Hz), including motion sensors (accelerometer for each axis) and rotation sensors (gyroscope providing roll, pitch and yaw rate),

- an odometer $(20 \mathrm{~Hz})$ measuring the distance traveled,

- a shaft encoder to measure the wheel steering angle.

Figure 1 also illustrates the vehicle coordinate frame $\mathcal{R}_{v}=$ $\left(X_{v}, Y_{v}, Z_{v}\right)$ where $Z_{v}$ denotes the altitude, and $\left(X_{v}, Y_{v}\right)$ plane is parallel to the ground.

The rigid transformation between the camera frames and the vehicle frame is know by experimentally measuring the

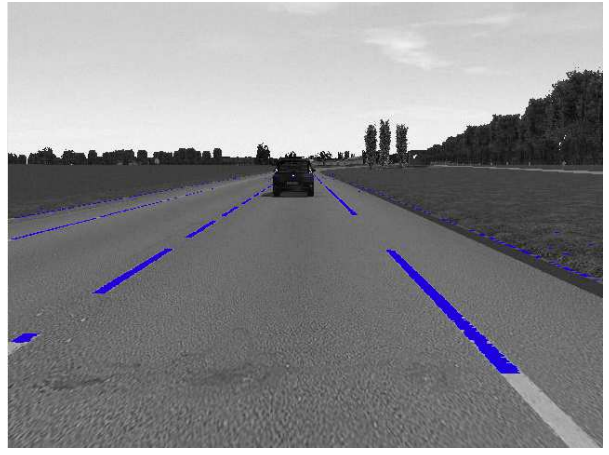

(a) Source image.

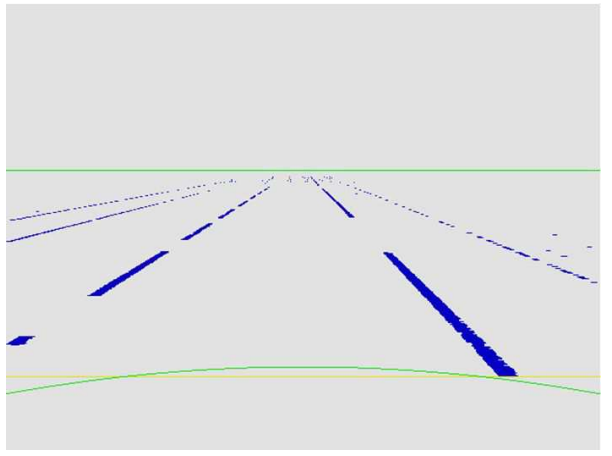

(b) Detected primitives.

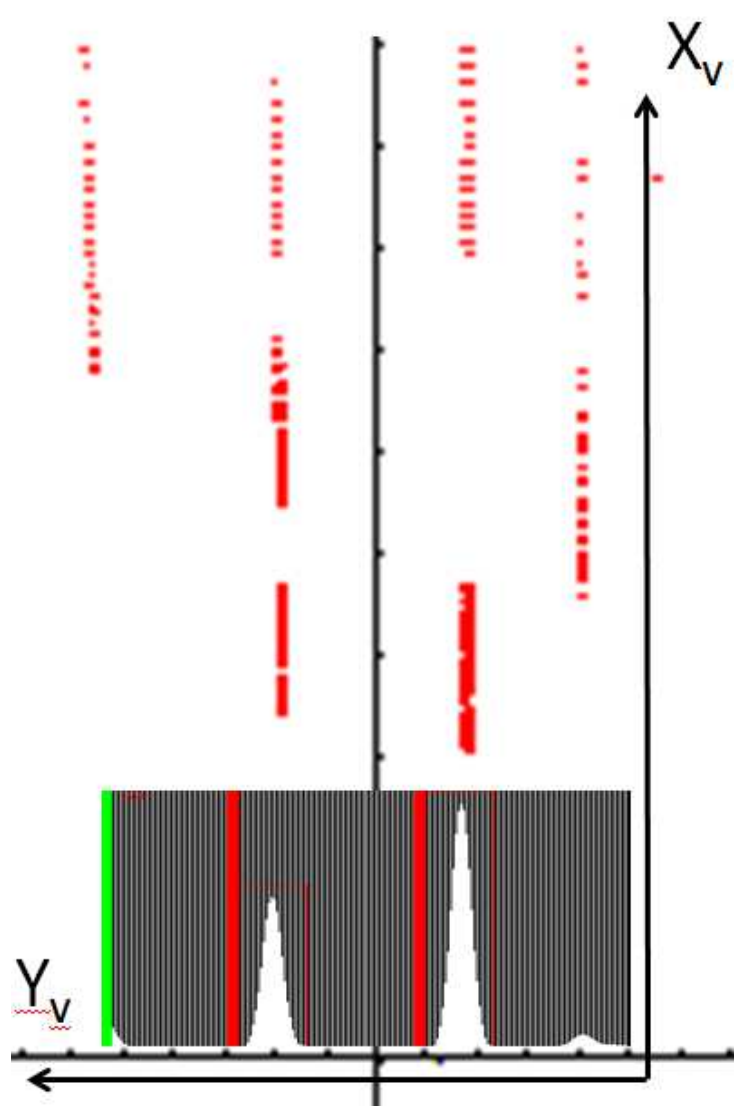

(c) Projection on the $\mathcal{R}_{v}$ frame.

Fig. 2. Lane marking primitive detection with a frontward camera.

extrinsic parameters for each camera. As the camera are calibrated, the intrinsic parameters are also known. 


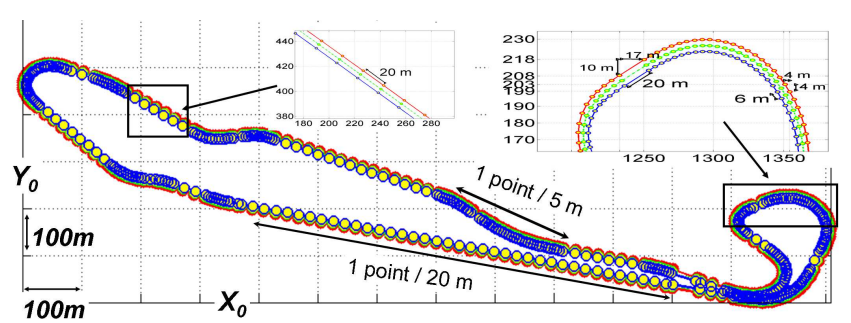

Fig. 3. Oversee of the Satory track map. The road lane is divided into band portions bounded by two triplet of points (yellow circles). Triplets of points are sampled every 4 to 20 meters depending on the curvature of the road

An other feature required for the proposed approach is a map with the precise location of the lane marking. Our experiments are run on the Satory (Versailles-France) test track which is a one way, $3.5 \mathrm{~km}$ long asphalt carriageway, with sharp bends, hairpin turns, as well as straight sections. A top-view of its map is displayed in Figure 3 . The total road width is 7 meters: it is a two-lanes way with three painted white lines defining the lane boundaries. Land surveys on the track provide a centimeter level accuracy of its road marking. The test track of Satory is a 2-lanes road precisely surveyed by professionals: coordinates of the road edges and the center line has been registered with a centimeter level accuracy. The resulting digital map is made of 380 triplets of points (1 sample every 5 meters on bends, 1 sample/20 meters on straight portions) also contains road features such as its curvature and type (straight, clothoid, arch). The sampling of the map is reduce to $10 \mathrm{~cm}$ by interpolation.

\section{Road Marking Detection using Two Lateral Cameras}

The road marking detector we developed is an adaptation of the work presented in [16], which will be summarized in this section for the sack of clarity. It is a three stages algorithm:

- road primitive extraction: this step determines which pixels belong to a lane marker. For each camera, this set of point is projected in the vehicle coordinate frame using the known intrinsic and extrinsic parameters.

- lane marking detection: the spatial distribution of extracted point on the $Y_{v}$ axis is analysed to determine the center of the potential marking lanes.

- lane shape estimation using a polynomial fitting. From this last step, two parameters are estimated: the vehicle yaw and its distance to the lane.

In order to improve the primitive extraction step, we proposed a cooperative combination strategy of two intensitybased extractors: the Median Local Threshold MLT and the Symmetric Local Threshold. Figure 2(a) illustrates the detected road primitives (in blue) superimposed on the source image.

Knowing the intrinsic and extrinsic camera parameters, it is possible to match a point $\mathrm{P}$ of the image with a real world point under the assumption that it is a point of the road. The set of pixels classified as road marking primitives of Figure 2,b) projected on the $\left(X_{v}, Y_{v}\right)$ plane of the vehicle

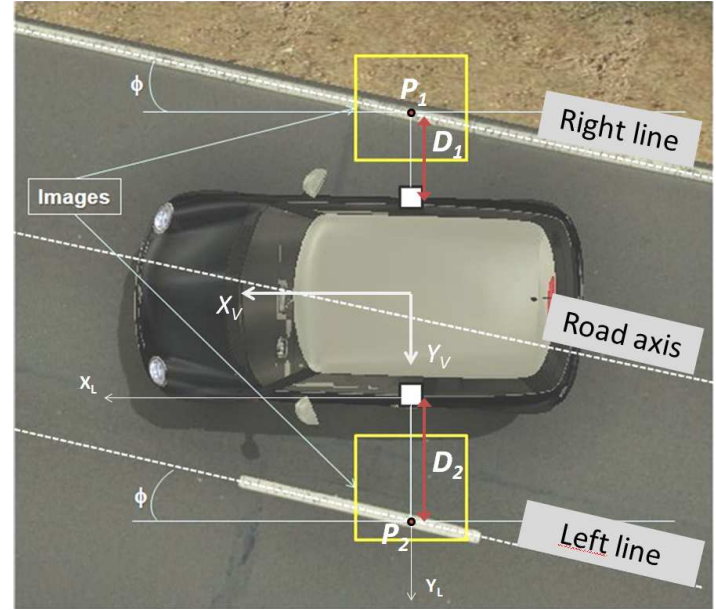

(a) Setup: top view
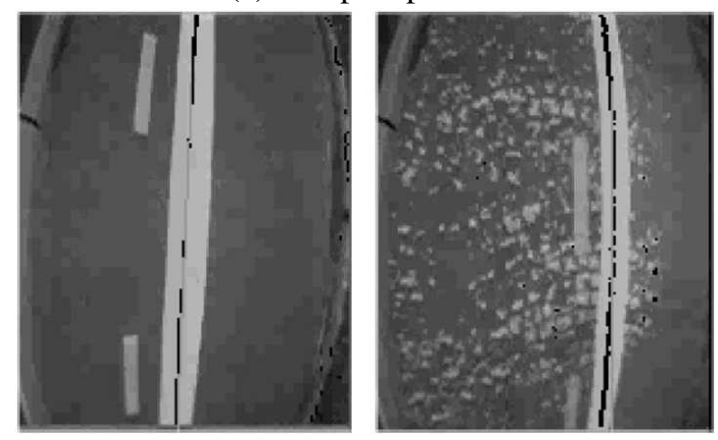

(b) Road line detection result

Fig. 4. (a) Analysis of lateral road marking. $\phi$ is the vehicle's yaw angle relatively to the road axis. $P_{1}$ and $P_{2}$ are the distance from the vehicle to the left and right road marking. (b) Samples of line detection on the Satory tracks.

frame is illustrated on Figure 2(c). A 1D projection on the $Y_{v}$ axis is performed using dynamic templates: it results in a 1D-histogram showing the spatial distribution of the road primitive along the $Y_{v}$ axis (Figure 2(c)). Within this distribution, clusters are identified using a tierce derivative and further selected based on mean belief, number of primitives, cluster standard deviation and length.

A robust poly-fitting is finally applied on each cluster so that the candidate lane is modeled by its equation on the $\left(X_{v}, Y_{v}\right)$ plane:

$$
y=a x^{2}+b x+c
$$

When the camera is directed frontward, the shape of the road especially its curvature introduces a lot of possible variations in the road marking appearance. Estimation of its parameters (curvature, slope, bank) is limited by the accuracy of the distance computation and can be sensitive to change in lighting conditions. In our apparatus, only the part of the marking the closest to the vehicle is being sensed as shown in Figure 4(a). In this configuration, detected primitives are less noisy and the effect of lighting variation is limited. Moreover, lane marking can be assumed to be locally linear. The distance $D_{1}$ (or $D_{2}$ ) from a camera to a lane as well as its orientation $\phi$ can be extracted from parameters $b$ and $c$ of Equation 11 $b=\tan \phi$ and $c=D_{1}$. The lateral distance 
ranges from 1 meter to 2.5 meters during our tests, while the vehicle traveled exclusively on the left lane.

\section{Ego-Vehicle Absolute Localization}

The ego-vehicle localization is estimated using an Extended Kalman Filter defined by the following equations:

$$
\begin{gathered}
X_{k+1}=f\left(X_{k}, U_{k}\right)+V_{k} \\
Y_{k+1}=h\left(X_{k+1}\right)+W_{k}
\end{gathered}
$$

$X_{k}=\left(x_{k}, y_{k}, \theta_{k}\right)^{T}$ is the vehicle state at time k. $Y_{k}$ is the measurement vector at time $\mathrm{k} . V_{k}$ and $W_{k}$ are the process and observation noises, assumed to be zero mean multivariate Gaussian noises with covariance $R_{k}$ and $Q_{k}$ respectively.

Regarding the prediction step, dead reckoning is performed by merging the proprioceptive data coming from the steering wheel coder (from which the actual front wheels steering radius $\theta_{w}$ is computed), the odometer measuring the distance $d_{k}$ traveled at time $t_{k}$, and the yaw rate $\dot{\theta}_{k}$ from the INS. The EKF is based on the widely used bicycle non linear model. An ellipse $\mathcal{E}_{k}$ representing the level of confidence on the localization can be build from the covariance matrix.

As both speed and direction must be accurately measured at all time, dead reckoning is subject to significant errors, as illustrated by Figure 5(a): each estimate of position being relative to the previous one, errors are cumulative [17]. To overcome this drift in time, other source of information are used, the most common being the GPS-receiver. The correction introduced by exteroceptive measurement such as the GPS data significantly improves the accuracy of the positioning estimate as shown by Figure 5 (b). However, GPS data are also subject to bias (see [18]) and a drift in vehicle localization can still be found especially in case of hairpin turn as illustrated in Figure 6(a) where the EKF filter results in a localisation outside of the roadway. To reach a lane-level accuracy, we propose to integrate the lane map information into the Kalman filtering.

\section{Lane Map Integration}

At this stage, the following capability are gathered:

- estimation of the distance from the vehicle to the left and right road marking of the lane,

- estimation of the vehicle localization using an extended Kalman filter.

The issue is now to adequately use these information to enhance the accuracy of the ego-localization. To build a bridge between these two approaches, we used the Satory cartography data.

Locally the track is approximated by a line segment. Using the estimated vehicle position, a point-to-segment based Map-Matching is performed to select a lane segment of the map. This segment is modeled by its polar equation featuring its polar coordinates $(\rho, \theta)$ :

$$
x \cos \theta_{\text {seg }}+y \sin \theta_{\text {seg }}-\rho_{\text {seg }}=0
$$

Image processing only provides local measurements. To obtain an absolute localization of the ego-vehicle, a cartography matching algorithm is applied. The distance $D_{i}\left(D_{1}\right.$ or

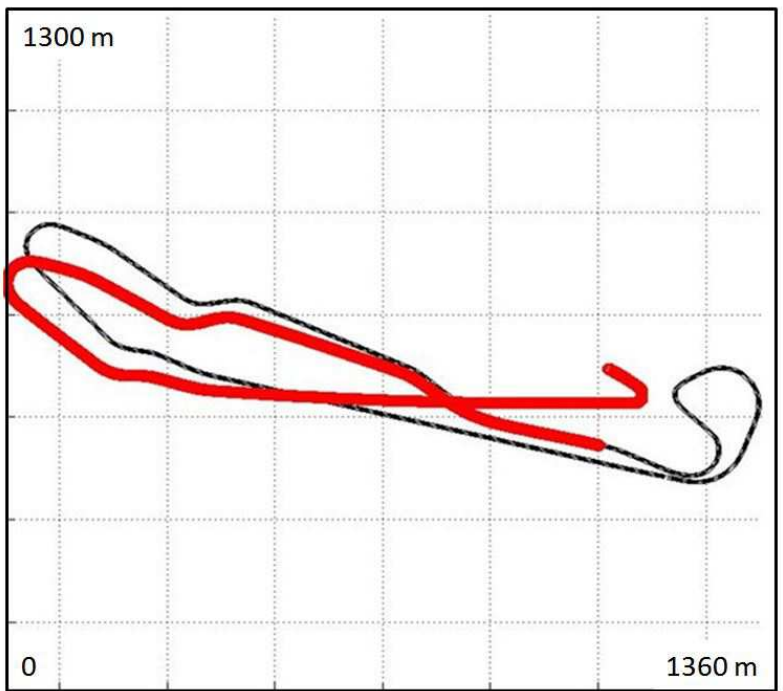

(a) Dead reckoning

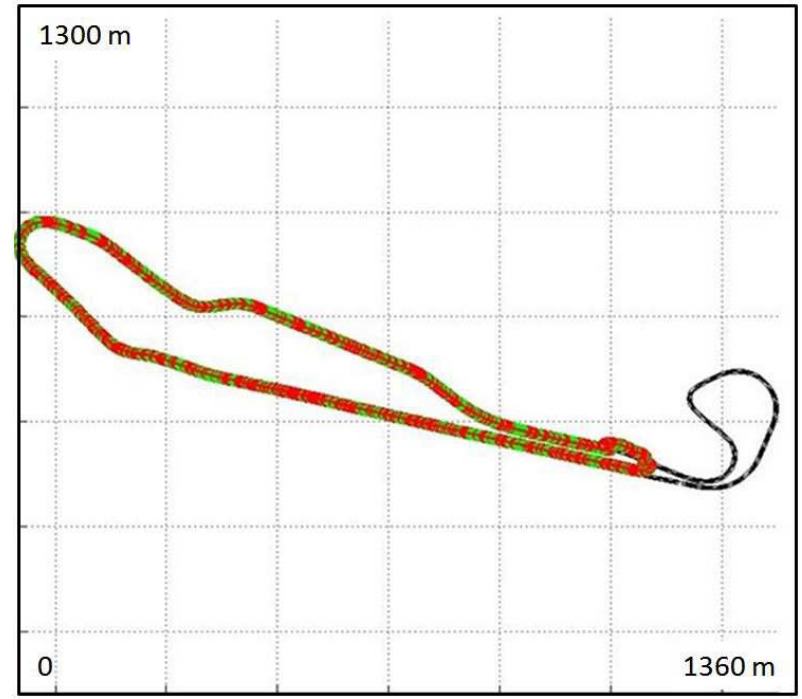

(b) IMU + GPS localization

Fig. 5. (a) Dead-reckoning with only an odometer and an inertial measurement unit. (b) Location with the use of the GPS for the filter's correction step.

$D_{2}$ see Figure 4(a)) between the camera and the marking is extracted from the lane marking equation $y=a x^{2}+\tan \phi x+D_{i}$ by image processing. The corresponding $P_{i}$ point coordinates are computed in the vehicle frame $\mathcal{R}_{v}$ :

$$
{ }^{v} P_{i}=\left[\begin{array}{c}
{ }^{v} X_{i} \\
{ }^{v} Y_{i}
\end{array}\right]=\left[\begin{array}{c}
{ }^{v} X_{c} \\
{ }^{v} Y_{c}
\end{array}\right]+D_{i}\left[\begin{array}{c}
\cos \theta_{c} \\
\sin \theta_{c}
\end{array}\right]
$$

where $\left({ }^{v} X_{c},{ }^{v} Y_{c}\right)$ are the coordinates of the camera center in the vehicle frame, $\theta_{c}$ the orientation of the camera frame in $\mathcal{R}_{g}\left(\theta_{c}=-\pi / 2\right.$ for the left camera and $\theta_{c}=\pi / 2$ for the right camera) and $D_{i}$ the distance of the closest point of the lane sensed by the camera.

The Jacobian of the measurement matrix from the polar measurement equation is:

$$
H_{k+1}=\left[\begin{array}{lll}
\frac{\partial h_{k+1}}{\partial x} & \frac{\partial h_{k+1}}{\partial y} & \frac{\partial h_{k+1}}{\partial \theta}
\end{array}\right]
$$




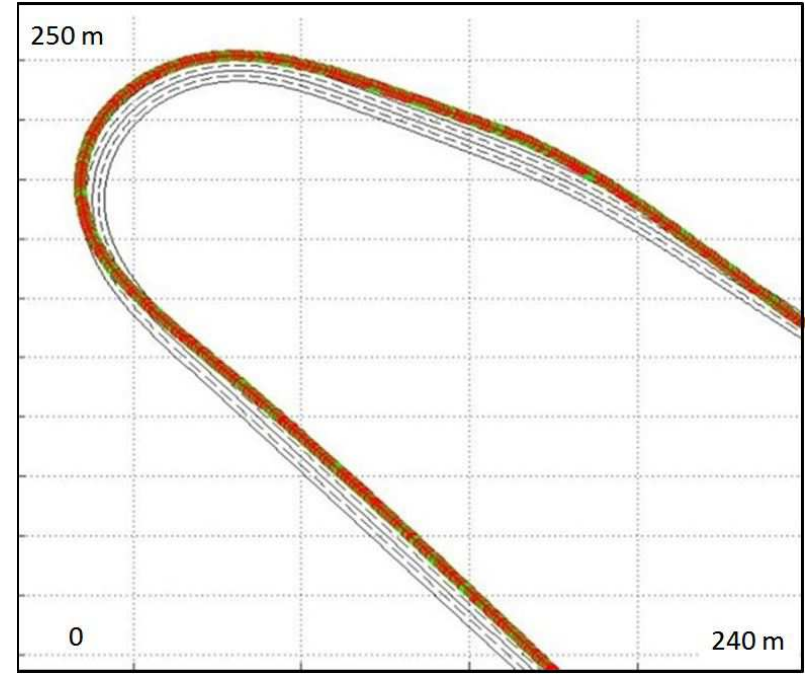

(a) Accuracy with GPS and inertial data

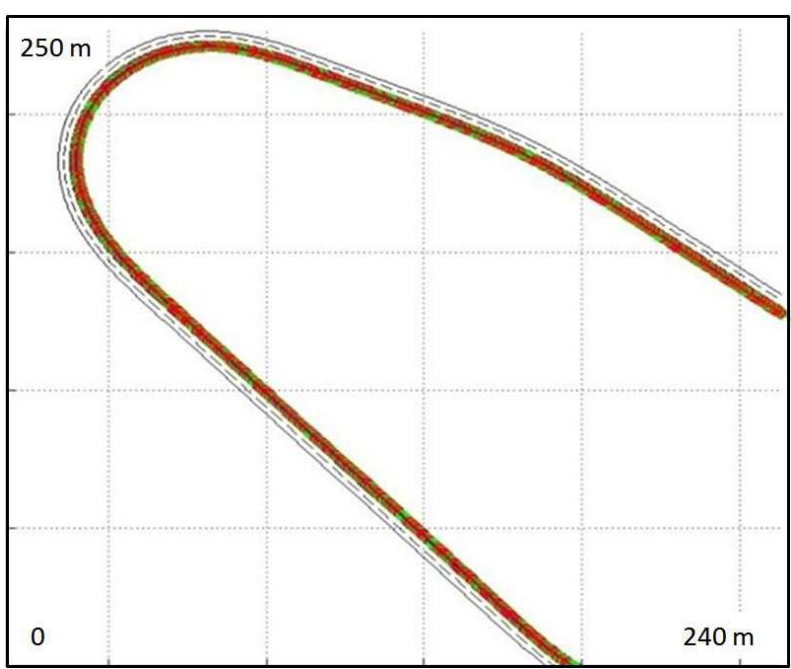

(b) Improvement using visual features

Fig. 6. (a) Error made by the Kalman filter with the merger of Odometer / INS / GPS. (b) Results obtained with the correction using lateral distance and accurate mapping.

with

$$
\begin{gathered}
\frac{\partial h_{k+1}}{\partial x}=\cos \theta_{\text {seg }} \\
\frac{\partial h_{k+1}}{\partial y}=\sin \theta_{\text {seg }} \\
\frac{\partial h_{k+1}=\quad}{\partial \theta}=\left(X_{c}+D_{i} \cos \theta_{c}\right) \sin \left(\theta_{\text {seg }}-\hat{\theta}_{k \mid k}\right) \\
-\left(Y_{c}+D_{i} \sin \theta_{c}\right) \cos \left(\theta_{\text {seg }}-\hat{\theta}_{k \mid k}\right)
\end{gathered}
$$

The coordinates of point $P_{1}$ and $P_{2}$ in the absolute frame $\mathcal{R}_{0}$ are then computed:

$$
\begin{aligned}
{\left[\begin{array}{c}
X_{P_{i}} \\
Y_{P_{i}}
\end{array}\right]=} & {\left[\begin{array}{c}
\hat{x}_{k \mid k} \\
\hat{y}_{k \mid k}
\end{array}\right]+\left[\begin{array}{cc}
\cos \hat{\theta}_{k \mid k} & \sin \hat{\theta}_{k \mid k} \\
-\sin \hat{\theta}_{k \mid k} & \cos \hat{\theta}_{k \mid k}
\end{array}\right]\left[\begin{array}{c}
{ }^{v} X_{c} \\
{ }^{v} Y_{c}
\end{array}\right] } \\
& +\left[\begin{array}{cc}
\cos \theta_{c} & \sin \theta_{c} \\
-\sin \theta_{c} & \cos \theta_{c}
\end{array}\right]\left[\begin{array}{c}
{ }^{v} X_{i} \\
{ }^{v} Y_{i}
\end{array}\right]
\end{aligned}
$$

$\hat{\theta}_{k \mid k}$ is the estimated yaw at time $\mathrm{k} .\left(\hat{x}_{k \mid k}, \hat{y}_{k \mid k}\right)$ are the estimated coordinates of the vehicle at time $\mathrm{k}$ (in the absolute frame). If $X_{P_{i}}$ and $Y_{P_{i}}$ measurement are unnoisy measurement, $P_{i}$ point belongs to matching segments of the map and the road marking. In this case, $X_{P_{i}} \cos \theta_{\text {seg }}+Y_{P_{i}} \sin \theta_{\text {seg }}-\rho_{\text {seg }}=0$, else $X_{P_{i}} \cos \theta_{\text {seg }}+Y_{P_{i}} \sin \theta_{\text {seg }}-\rho_{\text {seg }} \neq 0$. This error is used to update the positioning; the measurement error is then computed as:

$$
h_{k+1}=\left[\begin{array}{lll}
\cos \theta_{\text {seg }} & \sin \theta_{\text {seg }} & -\rho_{\text {seg }}
\end{array}\right]\left[\begin{array}{c}
X_{P_{i}} \\
Y_{P_{i}} \\
1
\end{array}\right]
$$

The Kalman gain is computed with matrix $H_{k}$ and enable to update the estimate:

$$
\hat{X}_{k+1 \mid k+1}=\hat{X}_{k+1 \mid k}+K_{k+1} h_{k+1}
$$

The covariance matrix is updated using $H_{k+1}$ and $R_{k}$.

\section{Results And Comments}

In order to validate this approach, we made a series of tests in simulated and real conditions. Results presented in this section come from a recording made in real conditions.

To properly see the benefits of the approach to an accurate location, we present 3 cases. First, we proposed an estimate location with only the use of an odometer and an inertial unit. Then we applied the updated estimates with GPS data. Finally we implemented the use of all data sources (Odometer, INS, GPS, and cameras).

With only the use of proprioceptive sensors, we see a drift of the filter due to the noise and bias of the inertial sensors. After traveling 30 meters, we already have $20 \mathrm{~cm}$ of error. After 100 meters, we get 1 meter of error. After 180 meters the error magnitude is 2 meters. This behavior is clearly observed in Figure 5(a). Once we implement the filter's correction phase with the use of GPS data, we find that the results greatly improve as shown in Figure 5(b). However, a zoom in on the nonlinear part (tight turn), illustrated in Figure 6(a) shows that the estimated position of the egovehicle is off the track while the vehicle is actually traveling on the left lane.

Using additional information relating to the lateral gap between the ego-vehicle and the roadsides provided by a lane detection algorithm, we obtain significantly better results (see Figure 6(b)). These results clearly show that the vehicle is properly located on the left lane. The whole test results are demonstrated in two online videos:

- GPS-IMU ego-localization without visual features: youtube.com/watch?v=N-0ANY7zqJs

- map-aided localization with lateral vision: youtube.com/watch?v=nSfc1UM7qIk

Figure 7 illustrates the difference in accuracy at several time of the test run. The samples in column (a) are estimated with the GPS and the IMU alone, while the samples of column 

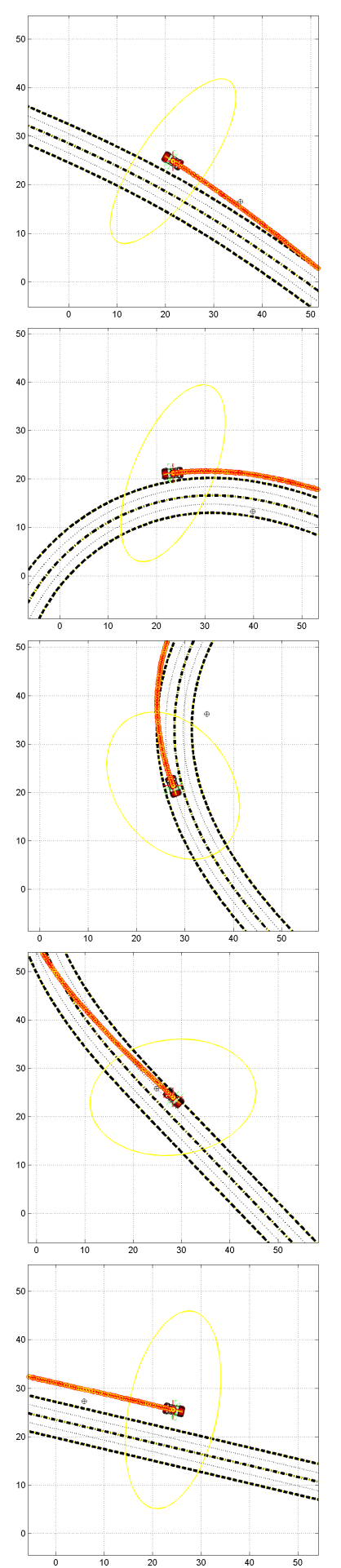

(a) GPS and IMU
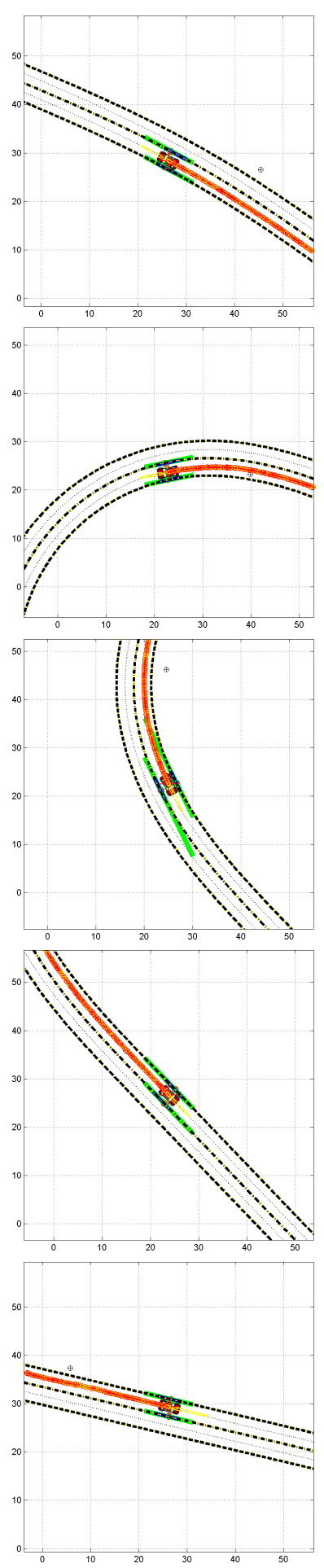

(b) GPS, IMU and lanes

Fig. 7. (a) Localization with the merger of Odometer / INS / GPS. (b) Localization by integration of lateral distance and accurate mapping. The confidence level on the estimation (yellow ellipse) is higher with visual features.

(b) also integrate the lane marking detection. During this test, the vehicle traveled on the left lane, and we can see that the GPS+dead reckoning approach is biased: it is mainly due to a high uncertainty on the GPS signal, which make the confidence ellipse $\mathcal{E}_{k}$ spatial extent quite important (yellow

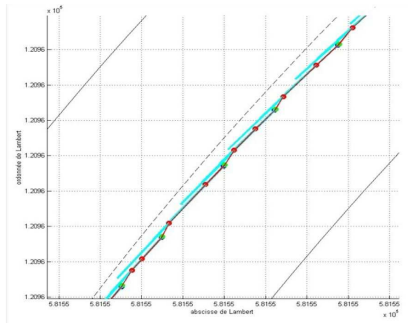

(a) Ego-lane level accuracy

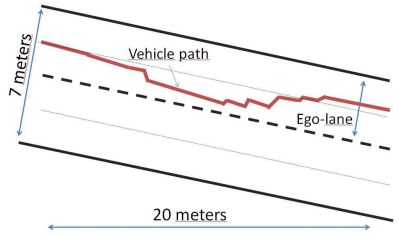

(b) Obstacle avoidance
Fig. 8. Level of accuracy inside the ego-lane.

ellipses). Map-aided localization makes a good estimate of the ego-lane, with a high confidence on the lateral position, the ellipse being stretched only in the longitudinal direction.

On this experiment, the lateral position estimation accuracy is less than $10 \mathrm{~cm}$ on average: Figure 8 (a) shows an example of trajectory drawn inside the ego-lane (the dotted line is the middle of the left lane). Figure 8 (b) illustrated the trajectory in the carriageway during an obstacle avoidance maneuver. However it is important to note that these good results are not only due to the quality of lane detection but also to the quality and accuracy of the mapping. It is obvious that mapping a large area with centimeter accuracy is heavy both in memory level resources than financial cost level.

The lane detection algorithm is also interesting because its accuracy is sufficient to identify the driver maneuvers. In addition, we do not actually use the runway heading information, which can be very useful to update the vehicle heading.

\section{Conclusion}

Currently our approach relies on the use of width markings information. However this information is not constant and can therefore generate inaccuracies. In future work, we propose to use a multi-lane detection algorithm with multicamera fusion, with a range limited to a maximum of 10 meter. Regarding the topology of the cameras, we will test several configurations (one front camera and one rear camera, 2 front cameras,...). What is interesting about our approach is that our traffic lane model is based on polynomial of degree $n$. Generally we limit the polynomials to order 2 to estimate the curvature, the direction and the lateral deviation of the marking. In this application dedicated to the location, only orientation information and lateral deviation are useful. In view of the results of our multi-lane detection algorithm, it is expected that the results will be really good.

\section{REFERENCES}

[1] Z. Tao, P. Bonnifait, V. Fremont, and J. Ibanez-Guzman, "Mapping and localization using gps, lane markings and proprioceptive sensors," in Proceedings of IEEE/RSJ International Conference on Intelligent Robots and Systems (IROS'13), 2013, pp. 406-412.

[2] T. Lefebvre, H. Bruyninckx, and J. D. Schutter, "Kalman filters for nonlinear systems: a comparison of performance," The International Journal of Control., vol. 77, no. 7, 2004.

[3] M. Norgaard, N. K. Poulsen, and O. Ravn, "Advances in derivativefree state estimation for nonlinear systems," in Revised edition of the technical report IMM-REP-1998-15, Technical University of Denmark, 2000. 
[4] R. Toledo-Moreo, M. Zamora-Izquierdo, B. Ubeda-Miarro, and A. Gomez-Skarmeta, "High-integrity imm-ekf-based road vehicle navigation with low-cost gps/sbas/ins," IEEE Transactions on Intelligent Transportation Systems, vol. 8, no. 3, pp. 491-511, 2007.

[5] A. N. Ndjeng, D. Gruyer, S. Glaser, and A. Lambert, "Low cost imu-odometer-gps ego localization with imm for unusual maneuvers," Information Fusion (Elsevier), vol. 12, no. 4, pp. 264-274, 2011.

[6] F. Gustafsson, N. Bergman, and U. Forsell, "Particles filters for positionning, navigation and tracking," IEEE Transactions on Signal Processing., vol. Special Issue on Monte Carlo methods for statistical signal processing, 2002.

[7] I. P. Alonso, D. F. Llorca, M. Gavilán, S. A. Pardo, M. Á. G. Garrido, L. B. Vlacic, and M. Á. Sotelo, "Accurate global localization using visual odometry and digital maps on urban environments," IEEE Transactions on Intelligent Transportation Systems, vol. 13, no. 4, pp. 1535-1545, 2012.

[8] M. Konrad, D. S. Nuss, and K. Dietmayer, "Localization in digital maps for road course estimation using grid maps," in Proceedings of IEEE Intelligent Vehicle Symposium (IV’2012), 2012, pp. 87-92.

[9] F. Schule, R. Schweiger, O. Lohlein, and K. Dietmayer, "Vehicle positioning on a digital map for road course prediction," in Future Active Safety Technology (FAST'2011), 2011.

[10] N. Mattern, R. Schubert, and G. Wanielik, "High-accurate vehicle localization using digital maps and coherency images," in IEEE Intelligent Vehicles Symposium (IV'10), 2010, pp. 462-469.

[11] T. Wu and A. Ranganathan, "Vehicle localization using road markings," in Proceedings of IEEE Intelligent Vehicle Symposium (IV'2013), 2013, pp. 1185-1190.

[12] H. Lategahn, M. Schreiber, J. Ziegler, and C. Stiller, "Urban localization with camera and inertial measurement unit," in Intelligent Vehicles Symposium, 2013, pp. 719-724.

[13] R. Toledo-Moreo, D. Betaille, F. Peyret, and J. Laneurit, "Fusing gnss, dead-reckoning, and enhanced maps for road vehicle lane-level navigation," IEEE Journal of Selected Topics in Signal Processing, vol. 3, no. 5, pp. 798-809, 2009.

[14] A. Schindler, "Vehicle self-localization with high-precision digital maps," in Proceedings of IEEE Intelligent Vehicle Symposium (IV'2013), 2013, pp. 141-146.

[15] S. Nedevschi, V. Popescu, R. Danescu, T. Marita, and F. Oniga, "Accurate ego-vehicle global localization at intersections through alignment of visual data with digital map," IEEE Transactions on Intelligent Transportation Systems, vol. 14, no. 2, pp. 673-687, 2013

[16] M. Revilloud, D. Gruyer, and E. Pollard, "An improved approach for robust road marking detection and tracking applied to multi-lane estimation," in Proceedings of IEEE Intelligent Vehicle Symposium (IV'13), 2013, pp. 783-790.

[17] F. Zhang, Simon, Chen, Buckl, and Knoll, "Cumulative error estimation from noisy relative measurements," in IEEE Conference on Intelligent Transportation Systems (ITSC'13), 2013, pp. 1422-1429.

[18] K. Jo, K. Chu, and M. Sunwoo, "Gps-bias correction for precise localization of autonomous vehicles," in Proceedings of IEEE Intelligent Vehicle Symposium (IV'2013), 2013, pp. 636-641. 